\title{
Clinical experience of somatostatin for the treatment of severe posthepatectomy liver failure
}

\author{
Hye-Sung JO, Young-Dong YU, Dong-Sik KIM*
}

Department of Surgery, Korea University College of Medicine, Seoul, Korea

Introduction: Posthepatectomy liver failure (PHLF) is a major cause of morbidity and mortality after major liver resection. Postoperative excessive portal pressure could cause shear stress to the small remnant liver leading to a PHLF. This study aimed to report the clinical experience of somatostatin for portal modulation in patients with severe PHLF.

Methods: This retrospective study enrolled 15 patients who received somatostatin for the treatment of PHLF between 2016 and 2019. When the patients fulfilled the $50-50$ criteria (serum bilirubin $>2.9 \mathrm{mg} / \mathrm{dL}$ and prothrombin time $<50 \%$ ) on or before postoperative day 5 , somatostatin $(3.5 \mu \mathrm{g} / \mathrm{kg} / \mathrm{h})$ was administered by continuous infusion. The discontinuation criteria were as follows: serum bilirubin $<2 \mathrm{mg} / \mathrm{dL}$ and prothrombin time $\geq 50 \%$. Prospectively collected clinical characteristics, laboratory tests, postoperative morbidity and mortality were evaluated.

Results: The study cohort consisted of 8 patients with hepatocellular carcinoma, 6 with cholangiocarcinoma, and 1 with colon cancer liver metastasis. Seven patients (46.7\%) had underlying liver cirrhosis, and 14 (93.3\%) underwent major hepatectomy. The median start time of somatostatin was postoperative day 1 (range, 1-19), and the median duration of administration was 9 days (2-29 days). There was no obvious side effects or hypersensitivity related to the somatostatin. The median hospital stay was 37 days (21-249 days). The 30 -day and 90 -day mortality were both $6.7 \%$ (1 of 15 patients).

Conclusions: Administration of somatostatin in the early postoperative period is considered to be effective for the treatment of PHLF. Further prospective comparative clinical trials are needed to validate this finding. 